\title{
Wireless Telemetry System for a SAW Based Microvalve
}

\author{
Ajay C. Tikka ${ }^{a, c}$ Said F. Al-Sarawi ${ }^{a, c}$ Behnam Jamali ${ }^{a, c}$ and Derek Abbott ${ }^{a, b, c}$ \\ ${ }^{a}$ Centre for High Performance Integrated Technologies and Systems (CHiPTec), The \\ University of Adelaide, SA 5005, Australia; \\ ${ }^{b}$ Centre for Biomedical Engineering, The University of Adelaide, SA 5005, Australia; \\ ${ }^{c}$ School of Electrical \& Electronic Engineering, The University of Adelaide, SA 5005, Australia
}

\begin{abstract}
Inductively coupled RF telemetry is an optimal method for both power supply and data transmission in long term artificial implants due to small size, high reliability, and extended life span of the device. In this research, we propose the use of the same technique for secure remote interrogation and powering of a human implantable, Surface Acoustic Wave (SAW) correlation based, passive microvalve. This is carried out by interrogating the microvalve with a Barker sequence encoded BPSK signal. In this paper we present the development of a FEM model for the derivation of the induced voltage on a miniature $(2.5 \times 2.5 \times 1 \mathrm{~mm})$, inductively coupled, biocompatible spiral antenna/coil, interrogated by a $7.5 \times 7.5 \times 0.2 \mathrm{~cm}$ spiral antenna/coil in the near field. The amount of power transferred at a $30-160 \mathrm{MHz}$ range was derived using the $\mathrm{S}_{21}$ coupling response when the two antennas are separated by a human body simulant of $5 \mathrm{~cm}$ depth. Furthermore, the effect of varying magnetic coupling on the induced voltage, due to the misorientation of coils/antennas is analysed.
\end{abstract}

Keywords: Surface Acoustic Wave (SAW) correlator, Finite Element Method (FEM).

\section{INTRODUCTION}

The rapid advances made in development of novel Microelectromechanical systems (MEMS) with bio-compatible materials have expanded their application domain in modern medicine for the deployment of implantable devices as diverse as pacemakers, retinal and cochlear implants, physiological recording devices, and drug delivery devices. ${ }^{1-3}$ Most of these devices are powered through batteries or wires, thus restricting their mobility, compactness and lifespan. Moreover, the development of a remotely powered and securely interrogated microactuator has not been fully exploited. Hence, a wireless controlled, Surface Acoustic Wave (SAW) correlator driven, passive microvalve is highly desirable. Numerical applications can be envisaged as such a device is light, reliable, chemically stable, and not limited by the lifetime of the battery. However, designing a telemetry system for such a microvalve is challenging due to the restriction on the receiving antenna size, impact of the lossy human tissue on the attenuation and antenna efficiency degradation.

Wireless transfer of power based on inductive coupling has been extensively investigated in the past..$^{4,5}$ However, most of these systems are incompatible with the proposed microvalve due to their large physical dimensions. Hence, there is a need to custom design a receiver coil with high coupling sensitivity for the reception of sufficient power. Furthermore, the common approach of treating the receiver and transmitter coils separately can not be applied to the current design. This is because the generation of magnetic field pattern and the power coupling through the media representing human tissue are significant for the design optimisation. To realise the inductive coupled telemetry system accurately, the receiver coil/antenna is solved simultaneously with the transmitter coil/antenna in the presence of human body simulant using 3-dimensional, high frequency electromagnetic, FEM modelling. The $\mathrm{S}_{12}$ response of the coils is analysed to figure out an adequate transmitterreceiver coil layout and to maximise the induced voltage at the implanted coil through the optimisation of magnetic coupling. The investigation of the variation in power transfer capabilities due to the changes in the

Further author information: (Send correspondence to Ajay Tikka)

Ajay Tikka: E-mail: ajay.tikka@adelaide.edu.au

Said Al-Sarawi: E-mail: alsarawi@eleceng.adelaide.edu.au

Biomedical Applications of Micro- and Nanoengineering IV and Complex Systems, edited by Dan V. Nicolau, Guy Metcalfe Proc. of SPIE Vol. 7270, 727018 - (C) 2008 SPIE · CCC code: 1605-7422/08/\$18 - doi: 10.1117/12.810602 
orientation of the coils is important for moving loads. This is carried out by tilting the transmitter coil by $60^{\circ}$ and analysing the drop in induced voltage due to reduced flux linkage between the misaligned coils.

In this paper, the FEM modelling and simulation of a 3-dimensional, bio-compatible, inductive power transmission link for a wireless controlled microvalve is presented. In Section 2, we discuss briefly the transmitter receiver architecture of the microvalve and the requirements placed on the power transmission link. Then in Section 3, we present the inductive link design, where the dimensions of the structure and materials are discussed. The simulation results comprising the quality factor, effective inductance, mutual coupling response, and induced voltage in the frequency domain are outlined in the section 4 . Furthermore, the variation of the power transfer capabilities due to the misorientation of the antennas is also discussed in the same section.

\section{WIRELESS POWERED MICROVALVE}

A brief description of the SAW correlation based microvalve telemetry system, as shown in the Fig. 1, is provided here. A SAW correlator is a passive pulse compression filter that operates through the correlation of phase shifts in the transmitted RF signal. It consists of an input IDT and a coded output IDT deposited on top of a piezoelectric substrate. The input IDT transduces the coded input RF signal into an acoustic wave. The electrodes of the output IDT are phase coded during construction in such a way that the correlator converts the correctly phase coded acoustic wave to a RF modulated electrical pulse. A more detailed description of the SAW correlator and microvalve operation was presented in Ref. [6].

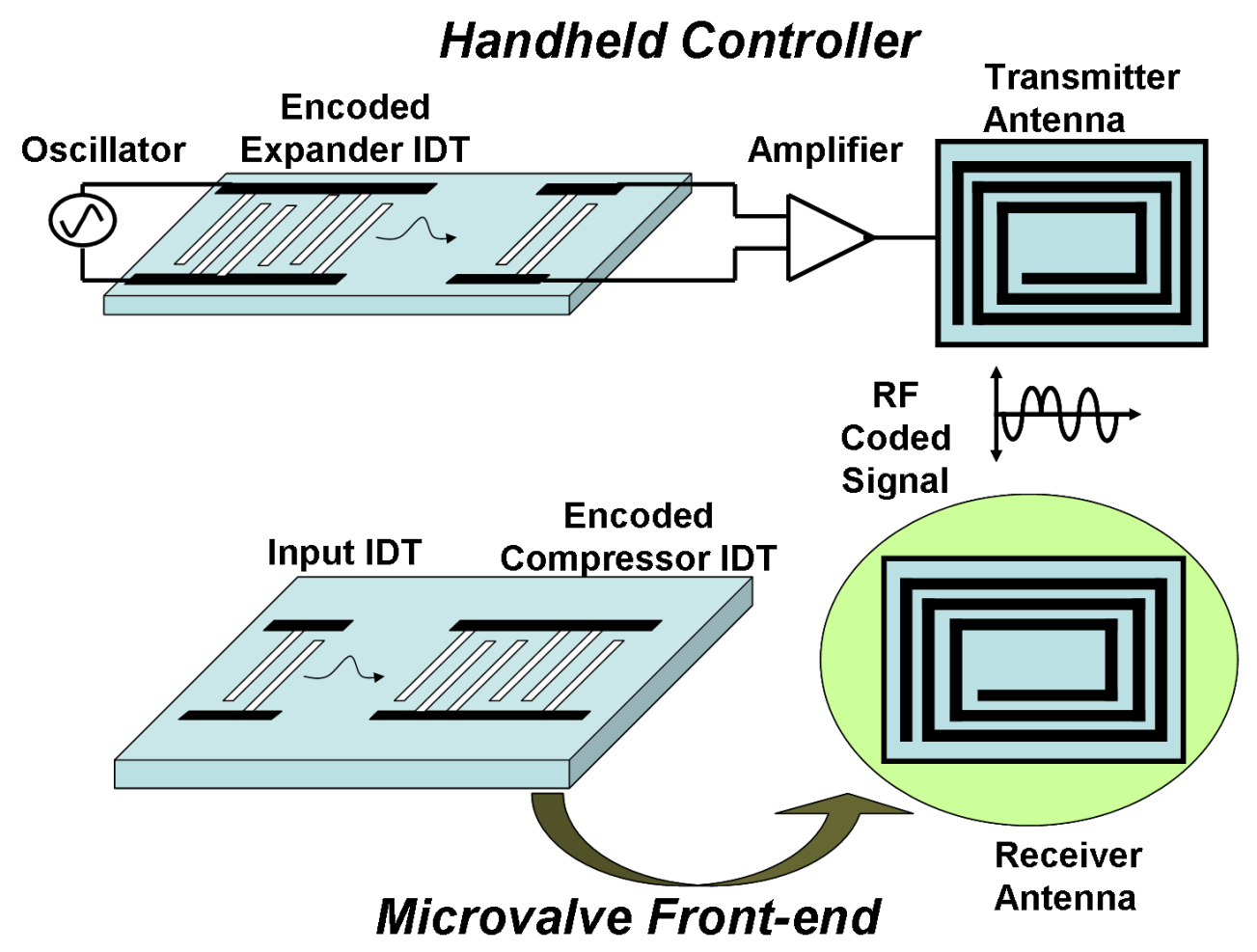

Figure 1. SAW correlator based microvalve transmitter receiver configuration. The hand-held controller generates a RF coded signal that triggers the microvalve when there is code match.

A coded SAW based communication system consists of an expander IDT in the hand-held controller and a compressor IDT in the implanted receiver. In the hand-held controller, a narrow pulse or a sinusoidal waveform is fed to the expander IDT to generate a coded acoustic signal depending on the geometry of the expander IDT. These acoustic waves propagate through the substrate to the transmitting IDT, which transforms these coded 
acoustic waves to electrical coded RF signals. The output from the transmitting IDT is fed to an amplifier, to strengthen the signal, and then to a transmitting antenna/coil. The front-end of the implanted microvalve consists of a correlator with its input IDT connected to a receiving antenna/coil to intercept the transmitted coded RF signal. The receiver coil is printed on the bottom surface of the correlator to ensure optimum space utilisation. The expander in the transmitter is an exact replica of the compressor/coded IDT of the correlator. The coding of the expander and compressor determines the autocorrelation function performed by the correlator. The microvalve will actuate only when the code of the interrogating RF pulse matches with the embedded code in the output IDT. The preferred coding scheme is a Barker sequence, and more details can be found in Ref. [7].

As the hand-held controller and the implanted microvalve are separated by a short distance, usually $5 \mathrm{~cm}$, establishing an inductive link using magnetic coupling is the preferred option. The requirements placed by the implanted microvalve on the receiver coil have to be analysed to design an effective inductive link with optimum power transfer. The dimensions of the implanted coil and the operating frequency are determined by the SAW correlator in the microvalve. As the SAW correlator is designed to operate at $50-100 \mathrm{MHz}$ with a physical dimension of $3 \times 3 \mathrm{~mm}$, the same limitations are placed on the receiver coil. The design of an inductive powering system which is complaint with the above mentioned specifications is discussed in the next section.

\section{INDUCTIVE LINK DESIGN}

The modelling of inductive coupling between the transmitter and receiver coil is mathematically equivalent to the resolution of the electromagnetic field equations for a given excitation. The Finite Element Method (FEM) is one of the most accurate methods of modelling the inductive link as the complete set of electromagnetic field equations is solved at radio frequencies in a 3-dimensional environment. Thus, an accurate evaluation of the effect of lossy human tissue, radiation and parasitic reactance is possible with this versatile technique. Moreover, the strong dependence of the loosely coupled power transmission link's performance on the trace metal thickness, line width, substrate material, number of turns, spacing between turns, impedance of the load, and the operating frequency provides a realistic prediction of the telemetry systems response subjected to a RF signal excitation. A 3-dimensional inductive link modelling was carried out using HFSS; a commercially available electromagnetic finite element package. The system, as shown in the Fig. 2, comprises of a transmitter antenna/coil and a biocompatible receiver antenna/coil separated by a human tissue simulant.

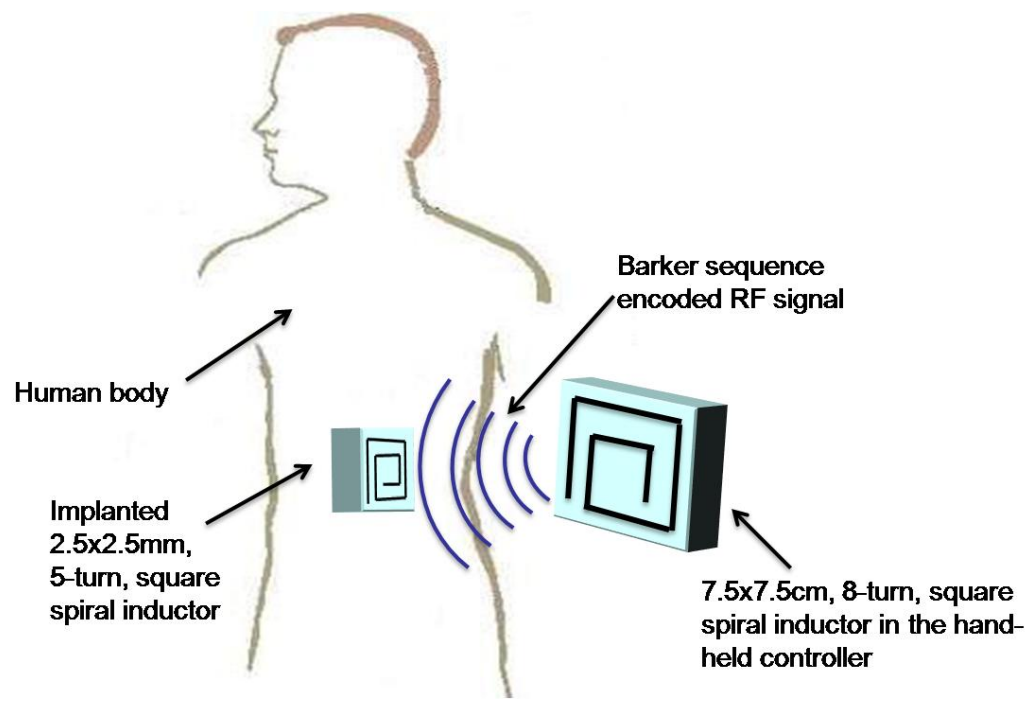

Figure 2. Inductive powering system of a wireless microvalve with an implanted coil/antenna and a transmitter coil/antenna (Note: Figure not to scale). 


\subsection{Transmitter Coil/Antenna}

The various inductive powering design approaches in the literature seem not to fully address the modelling of transmitter coil and the receiver coil simultaneously. A common practice has been to ignore the generation of magnetic field pattern by treating the two coils separately or to make use of empirical rules ${ }^{8,9}$ However, for the current application, where high coupling sensitivity and efficiency are needed, modelling and optimization of the complete system in the presence of human body simulant is necessary. As the transmitter coil is placed outside the body, there are fewer restrictions placed on its size. The efficiency of the power transfer is determined by the mutual inductance between the coils, which in turn is a function of the self inductances of the individual coils. Hence, a transmitter coil with 8-turn spiral pattern and an outer dimension of $7.5 \times 7.5 \mathrm{~cm}$ was chosen to ensure high self inductance. Gold, with a thickness of $10 \mu \mathrm{m}$, is used to model the coil on a polyamide substrate with a relative permittivity of 4.3. Both the line width of the coil and the spacing between the turns was set to $1.5 \mathrm{~mm}$.

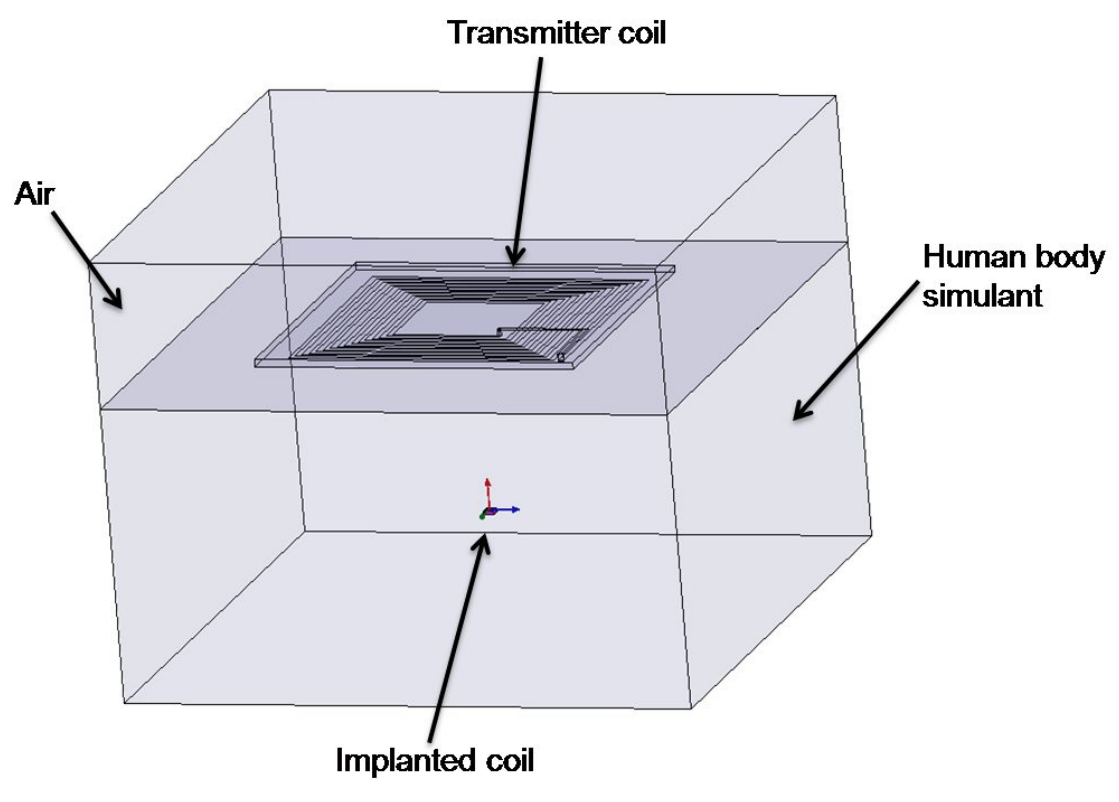

Figure 3. Simulation setup for the design of the inductive coupled telemetry system.

\subsection{Receiver Coil/Antenna}

The design of an implantable antenna determines the radiation dependent mutual inductance and power transfer efficiency of the system and therefore is a critical step in the modelling of the telemetry link. As the physical dimensions of the implanted coil are restricted to those of the SAW correlator, which in this case is $3 \times 3 \mathrm{~mm}$, there is a limit placed on the self-inductance of the coil and hence the mutual inductance. This problem can be tackled by enhancing the quality factor of the coil, which is carried out by increasing the coil thickness and reducing the spacing between the turns. The receiver coil comprises of a $2.5 \times 2.5 \mathrm{~mm}, 5$-turn square spiral, $15 \mu \mathrm{m}$ thick gold coil printed on a polyamide substrate of thickness $1 \mathrm{~mm}$. The line width of the coil is $50 \mu \mathrm{m}$ and the spacing between the turns is $25 \mu \mathrm{m}$. The choice of the material is guided by an aim to make the implanted coil bio-compatible. Furthermore, the correct application of boundary conditions is important to ensure a realistic modelling of the system. Hence, to capture the dynamic behavior of the implant and to account for the strong attenuation and multiple reflections of the electromagnetic waves in a biological environment the implanted coil is surrounded, and separated from the transmitter coil, by a human body simulant. The best practice to represent the average body properties is to consider the electrical properties of the the human body simulant as $2 / 3$ those of pure muscle. ${ }^{10}$ Hence, the receiver antenna is placed in a $16 \times 16 \times 80 \mathrm{~cm}$ block of $2 / 3$ human muscle, as shown 
in the inductive link FEM model in Fig. 3. The simulation results of this inductive link, where the separation between the coils is $5 \mathrm{~cm}$, are presented in the next section.

\section{RESULTS}

The 3-dimensional FEM simulations of the inductive link, the results of which are discussed in this section, are driven by the goal to determine an optimum layout for the transmitter and implanted coils in the context of the operating environment. This is carried by analysing the frequency dependent behavior of the effective inductance, quality factor of the individual coils, the $\mathrm{S}_{12}$ coupling response, and induced voltage of the complete telemetry system.

\subsection{Quality Factor and Effective Inductance}

Our main objective is to model a inductive telemetry link with high coupling efficiency, that can power a miniature wireless microvalve in a secure manner, through the optimisation of design parameters. Two important design parameters that influence the power transfer capabilities of such a link are the quality factor and effective inductance of the coils. Here, the quality factor (Q) is the ratio of the magnetic energy stored to the power dissipated and the self inductance of the inductor along with the associated parasitic capacitance $\left(\mathrm{L}_{\mathrm{eff}}\right){ }^{11}$ is given by

$$
\begin{aligned}
Q & =-\frac{\operatorname{Im}\left(\mathrm{Y}_{11}\right)}{\operatorname{Re}\left(\mathrm{Y}_{11}\right)}, \\
L_{\mathrm{eff}} & =-\frac{1}{\omega \operatorname{Im}\left(\mathrm{Y}_{11}\right)},
\end{aligned}
$$

where $Y_{11}$ is the short-circuit input admittance of the coils and $\omega$ is the angular frequency.

To improve the mutual coupling, it is desirable to have a high value of inductance for the two coils. Furthermore, the quality factor of the implanted coil should be high, to minimise the RF power losses, and the transmitter coil should be low, to compensate for the misalignment of coils.

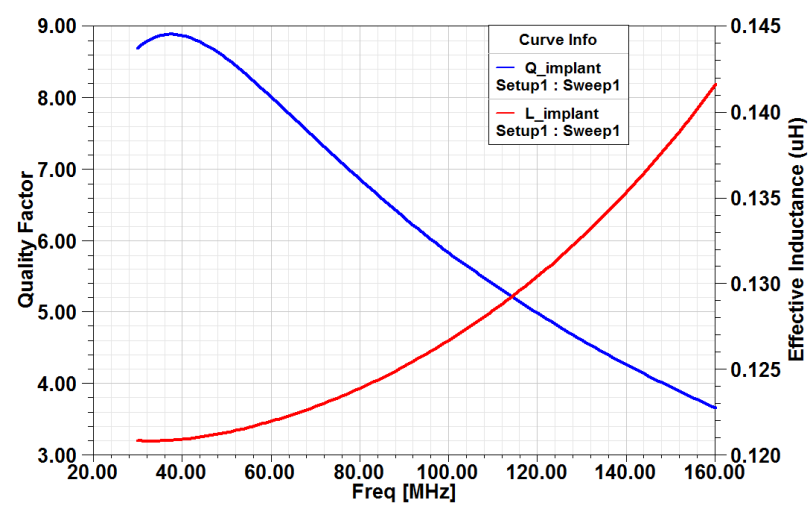

(a)

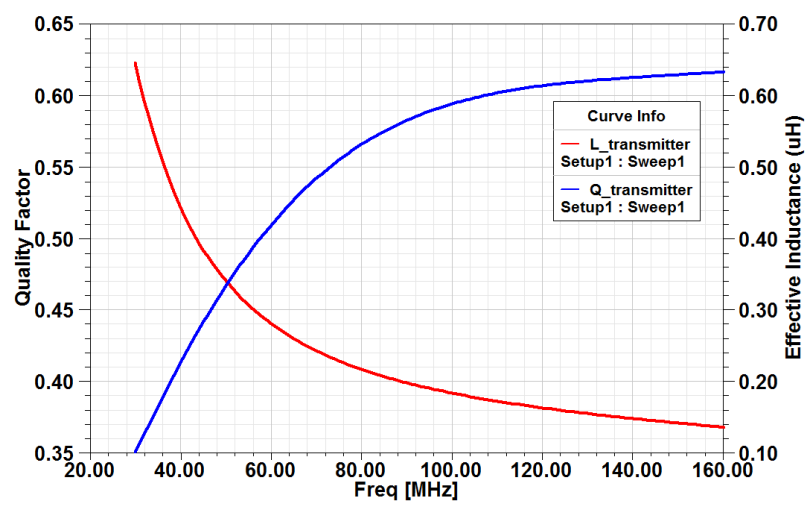

(b)

Figure 4. Quality factor $(\mathrm{Q})$ and inductance $\left(\mathrm{L}_{e f f}\right)$ of (a) Implanted coil. (b) Transmitter coil.

The effective inductance and quality factor of both the implanted and transmitter coils are computed from the admittance using the Eqn. (1) and (2), as shown in the Fig. 4. At $60 \mathrm{MHz}$, which is the operating frequency of interest from a SAW correlator point of view, the $\mathrm{Q}$ value of the receiver coil is 8 and the corresponding effective inductance is about $122 \mathrm{nH}$. At the same excitation frequency the $\mathrm{Q}$ value of the transmitter coil is low, as desired, at 0.56 and the effective inductance is $280 \mathrm{nH}$. 


\subsection{Mutual Coupling and Induced Voltage}

Depending on the type of inductive link, various methodologies can be used to analyse the coupling between the transmitter and receiver coils. The most frequently used ones are the transformer model and the mutual inductance model. ${ }^{12}$ The transformer model is ideal for closely coupled inductive link as the leakage inductance is ignored. However, the mutual inductance model considers both the coupling and leakage inductances and therefore is more suitable for weakly coupled inductive links. As the looses in the medium and the attenuation of the RF radiation are crucial for the accurate modelling of our telemetry system, the mutual inductance model is employed. Mutual inductance is a measure of the voltage induced in the implanted coil when the transmitter coil is excited by an alternating current. Fig. 5.(a) shows the $S_{21}$ response representing the coupling between the coils. The $S_{21}$ response is determined by the effect of the transmitter coil on the implanted coil, the intrinsic properties of the implanted coil, separation between the coils, transmitting medium and the frequency of operation. As can be seen from the Fig. 5.(a), the mutual coupling is maximum at $60 \mathrm{MHz}$. This coupling response is used to determine the induced voltage.

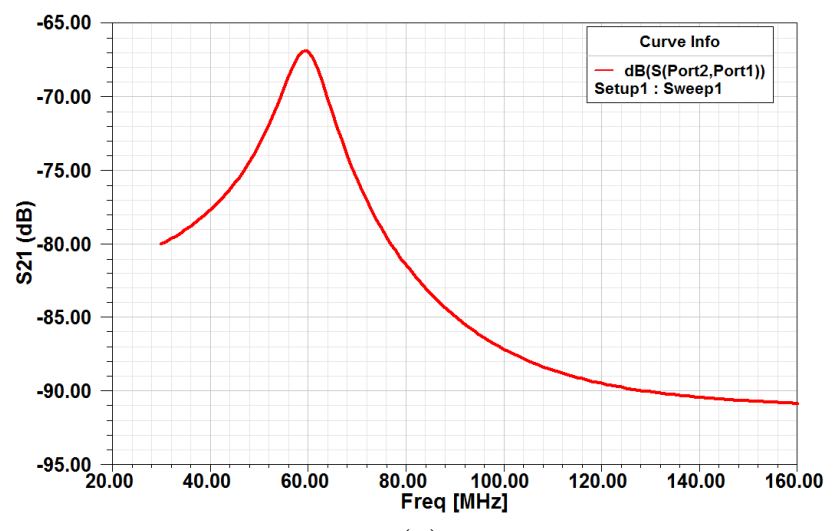

(a)

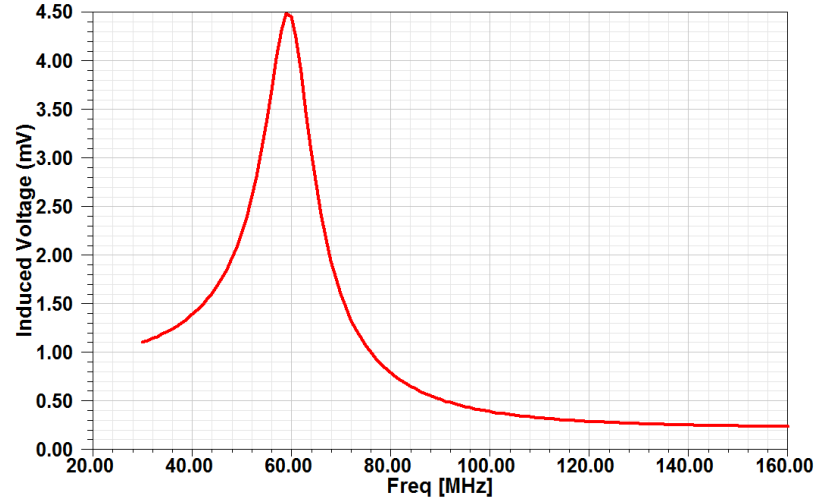

(b)

Figure 5. Coupling between the implanted and transmitter coil separated by a human body stimuli of depth $5 \mathrm{~cm}$ (a) $\mathrm{S}_{21}$ response. (b) Induced Voltage in the implanted coil when the transmitter coil is driven by $10 \mathrm{~V}(\mathrm{p}-\mathrm{p}) \mathrm{RF}$ signal.

Fig. 5.(b) shows the induced voltage response of the implanted coil, when the transmitter coil is driven by a $10 \mathrm{~V}(\mathrm{p}-\mathrm{p})$ input. At the operating frequency of interest, i.e. $60 \mathrm{MHz}$, a maximum voltage of magnitude $4.5 \mathrm{mV}$ is induced. The value of the induced voltage may seem quite low but is considered normal for this type of weak coupling in the presence of human body simulant. ${ }^{13}$ The above results reflect the accuracy of the telemetry system design for a given separation between the transmitter and the implanted antennas.

\subsection{Coil Misorientation}

A study of the wireless power transmission under varying coupling conditions is mandatory to accurately characterise the performance of the telemetry system. One such condition, which influences the efficiency of magnetic coupling in moving loads is the orientation of the coils. Any misorientation of coils results in a reduction in the induced output voltage, as the rate of change of magnetic flux is directly proportional to the induced voltage. For the above discussed simulation model, the transmitter antenna is tilted by $60^{\circ}$ and the variation in the power transfer capability was analysed.

The response of the misaligned inductive link is provided in the Fig. 6, while all other parameters are kept the same. By comparing this response, with the voltage response of the Fig. 5.(b), it is observed that there is a $56 \%$ drop in the induced voltage when the transmitter coil is tilted by $60^{\circ}$ at an operating frequency of $60 \mathrm{MHz}$. Thus, the analysis of the coil misorientation allows for the identification of the minimum driving power required to guarantee the operation of the microvalve. 


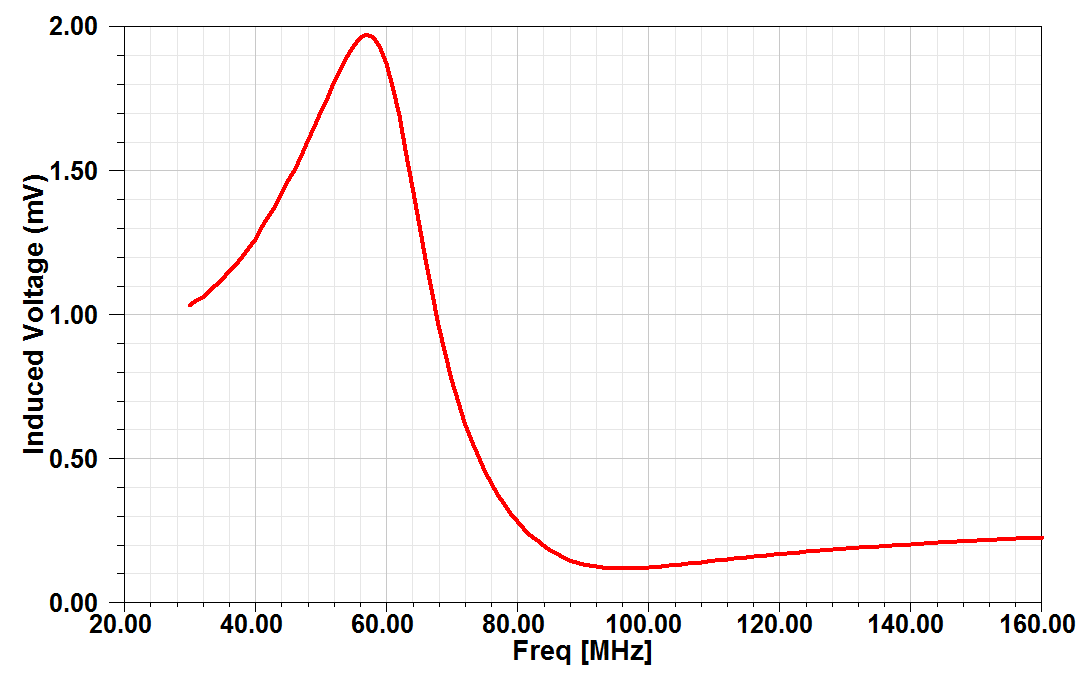

Figure 6. Voltage induced in the implanted coil when the transmitter coil is tilted by $60^{\circ}$.

\section{CONCLUSION}

The development of novel and innovative biomedical implants is placing new challenges on the existing wireless powering systems. This paper, using FEM modelling, addresses the issues of the implanted antenna miniaturisation and the near-field coupling between the hand-held controller and a wireless microvalve in the presence of a human body stimuli. The results discussed in this work include: $(i)$ Comprehensive FEM modelling of a 3 -dimensional, inductively coupled link comprising a $2.5 \times 2.5 \times 1 \mathrm{~mm}$ bio-compatible implanted antenna and a $7.5 \times 7.5 \times 0.2 \mathrm{~cm}$ transmitter antenna separated by a human body stimuli $(\mathrm{ii})$ analysis of the quality factor and inductance of individual coils (iii) observation of the mutual coupling $\left(\mathrm{S}_{21}\right)$ response and the induced voltage in the implanted antenna $(i v)$ analysis of difference in induced output voltage when there is a coil misorientation. Hence, FEM modelling provides an effective way of gaining a deeper understanding of the inductive link's response for various coupling conditions and ultimately the full optimisation of the telemetry system for an implantable microvalve.

\section{ACKNOWLEDGMENTS}

The authors would like to thank the Australian Research Council (ARC) and the School of Electrical and Electronics Engineering (University of Adelaide) for the funding and support for the project.

\section{REFERENCES}

1. P. Li, J. Shih, R. Lo, B. Adam, R. Agarwal, and S. Saati, "An electrochemical intraocular drug delivery device," in Proc. of 20th IEEE International Conference on Micro Electro Mechanical Systems, pp. 15-18, January 2007.

2. A. Grayson, R. Shawgo, Y. Li, and M. Cima, "Electronic mems for triggered delivery," in Advanced Drug Delivery Reviews, vol. 56, pp. 173 - 184, 2004.

3. S. Amer and W. Badawy, "An integrated platform for bio-analysis and drug delivery," in Current Pharmaceutical Biotechnology, vol. 6, pp. 57-64, February 2005.

4. H. Lu, C. Goldsmith, L. Cauller, and J. Lee, "MEMS-Based inductively coupled RFID transponder for implantable wireless sensor applications," in IEEE Trans. on Magnetics, vol. 43, pp. 2412-2414, June 2007.

5. P. Basset, A. Kaiser, B. Legrand, D. Collard, and L. Buchaillot, "Complete system for wireless powering and remote control of electrostatic actuators by inductive coupling," in IEEE Trans. on Mechatronics, vol. 12, pp. 23-31, February 2007. 
6. A. Tikka, S. Al-Sarawi, and D. Abbott, "Finite element modelling of SAW correlator," in Proc. of SPIE BioMEMS and Nanotechnology III conference, vol. 6799, p. 679915, December 2007.

7. A. Tikka, S. Al-Sarawi, D. Abbott, M. Wong, and J. Schutz, "Improving the security and actuation of wireless controlled microvalve," in Proc. of SPIE Smart Structures, Devices, and Systems conference, vol. 6414, p. 64140U, January 2007.

8. J. Kim and Y. Rahamat-Samii, "Planar inverted-F antennas on implantable medical devices: Meandered type versus spiral type," in Microwave and Optical Technology Letters, vol. 48, pp. 567-572, January 2006.

9. M. Theodoridis and S. Mollov, "Distant energy transfer for artificial human implants," in IEEE Trans. on Biomedical Engineering, vol. 52, pp. 1931-1938, November 2005.

10. P. Soontornpipit, M. Furse, and Y. Chung, "Design of implantable microstrip antenna for communication with medical implants," in IEEE Trans. on Microwave Theory and Techniques, vol. 52, pp. 1944-1951, August 2004.

11. I. Bahl, Lumped Elements for RF and Microwave Circuits, ch. 2. Artech House, 2003.

12. C. Wang, O. Stielau, and G. Covic, "Load models and their application in the design of loosely coupledinductive power transfer systems," in Proc. of International Conference on Power System Technology, vol. 2, pp. 1053-1058, December 2000.

13. R. Simons and F. Miranda, "Modeling of the near field coupling between an external loop and an implantable spiral chip antenna in biosensor systems," in Proc. of IEEE Antennas and Propagation Society International Symposium, pp. 1099-1102, July 2006. 